\title{
Rapid diagnosis of human prion disease using streptomycin with tonsil and brain tissues
}

\author{
Isabelle Quadrio ${ }^{1}$, Séverine Ugnon-Café ${ }^{1}$, Maryline Dupin² ${ }^{2}$, Guillaume Esposito ${ }^{1}$, Nathalie Streichenberger ${ }^{1}$, \\ Pierre Krolak-Salmon ${ }^{1}$, Anne Vital ${ }^{3}$, Jean-François Pellissier ${ }^{4}$, Armand Perret-Liaudet ${ }^{1}$ and Hervé Perron ${ }^{2,5}$
}

The use of streptomycin in the pathological prion protein $\left(\mathrm{PrP}^{\mathrm{sc}}\right)$ detection procedures represents a new and attractive way for diagnostic purpose. With this agent, western blot readily detected $\mathrm{PrP}^{\mathrm{sc}}$ in $263 \mathrm{~K}$ scrapie hamster and $\mathrm{C} 57 \mathrm{BI} / 6$ wild-type mice challenged with C506M3 scrapie strain. Our aim was to evaluate this new diagnosis procedure in the field of human transmissible spongiform encephalopathies (TSEs). First, we had confirmed the ability of streptomycin to precipitate PrPres from human brain of Creutzfeldt-Jakob disease (CJD) patient. Second, we compared the detection of $\mathrm{PrP}^{\text {res }}$ with streptomycin against three other protocols using other precipitations. Then we assessed PrPres detection with streptomycin in 98 brain tissue samples from various aetiologies of human TSEs and 52 brain samples from other dementia. Finally, we applied this protocol for tonsils examination of five patients suspected of variant CJD (v-CJD). Sensitivity and specificity obtained with the streptomycin protocol were both $100 \%$ on brain tissue. For tonsil tissues, $\mathrm{PrP}^{\text {res }}$ was clearly identified in the two post-mortem confirmed v-CJD cases, whereas no characteristic three-band pattern was seen in the three confirmed non-v-CJD samples. In this study, streptomycin demonstrated its efficiency to detect $\mathrm{PrP}^{\text {res }}$ both in the central nervous system and in the lymphoid tissue without practical difficulty and with rapid preparation. Because of its ability to act as a good agent for $\operatorname{PrP}^{\mathrm{Sc}}$ examination in different tissues, recovery of PrP ${ }^{\mathrm{sc}}$ in biological fluids using streptomycin should open further perspectives of applications in CJD diagnostics. Streptomycin effects in vivo might thus also be questioned.

Laboratory Investigation (2009) 89, 406-413; doi:10.1038/labinvest.2008.169; published online 2 February 2009

KEYWORDS: diagnosis; prion disease; streptomycin; tonsil; western blot

The normal or native prion protein, designated as $\operatorname{PrP}^{\mathrm{c}}$ for the cellular prion protein, is a widely occurring protein, the sequence of which is well conserved in mammals. ${ }^{1}$ Conformational changes in $\mathrm{PrP}^{\mathrm{c}}$ lead to propagation of the pathogenic prion protein ${ }^{~} \mathrm{PrP}^{\mathrm{Sc}}$ ' that is proteinase $\mathrm{K}(\mathrm{PK})$ resistant, at least for its core fragment, which is referred as 'resistant $\operatorname{PrP}$ ' $\left(\operatorname{PrP}^{\text {res }}\right)$. The $\operatorname{PrP}^{\text {sc }}$ accumulation in animals is associated with a wide range of diseases classified as 'transmissible spongiform encephalopathies' (TSEs). TSEs cover a series of transmissible neurodegenerative diseases, including scrapie in sheep, bovine spongiform encephalopathy (BSE) in cattle, chronic wasting disease in deer and elk, and Kuru and Creutzfeldt-Jakob disease (CJD) in humans. ${ }^{2}$ As represented by Gerstmann-Straüssler-Scheinker disease and fatal familial insomnia (FFI) in humans, TSE forms may also have genetic origins linked to mutations in the $\mathrm{PrP}$ gene that affect thermodynamics of the encoded protein in a spontaneous way favouring beta-sheet transconformation of $\operatorname{PrP}^{\mathrm{c}}$ to $\operatorname{Pr} \mathrm{P}^{\mathrm{sc}}$. Consequently, a significant probability to evolve towards pathogenic propagation during the lifetime exists in individuals affected by such genetic mutations in their inherited genotype. ${ }^{3,4}$ Coincidently with the outbreak of BSE epidemics and the confirmed transmission by food products, a new form of human CJD appeared with original histopathological and biochemical characteristics of $\operatorname{PrP}^{\mathrm{sc}}$, which was named 'new variant CJD' (v-CJD). ${ }^{5,6}$ It is now admitted that $\mathrm{v}$-CJD originates from the bovine BSE epidemics by passage to humans through alimentation. ${ }^{7,8}$

\footnotetext{
${ }^{1}$ Neurobiology Department, Groupement Hospitalier Est, Hospices Civils de Lyon, Lyon, France; ${ }^{2}$ BioMérieux Advanced Technology Department, Neurological Disease Unit, BioMérieux SA, Marcy l'Etoile, Lyon, France; ${ }^{3}$ Neuropathology Department, Victor Ségalen-Bordeaux 2 University, Bordeaux, France and ${ }^{4}$ Neuropathology Department, Medical University of Marseille, Marseille, France

Correspondence: Dr H Perron, BioMérieux Advanced Technology Unit, R\&D Neurological Diseases, Geneuro, 14 Chemin des Aulx, Plan-Les Ouates, Geneva 1228, Switzerland.

E-mail: hp@geneuro.com

${ }^{5}$ Current address: Geneuro, 14 Chemin des Aulx, 1228 Plan-Les Ouates, Geneva, Switzerland

Received 25 July 2008; revised 15 October 2008; accepted 16 October 2008
} 
The slow development and appearance of symptoms in cattle infected with BSE, with average incubation periods over 4-6 years, have greatly hindered the development of epidemic models as well as the reality of a passage to humans, itself hindered by an even longer period of incubation, which may last for decades. ${ }^{9}$ Indeed, the antigenic $\operatorname{PrP}^{\text {sc }}$ load is generally thought to increase progressively after infection, in lymphoid and peripheral nervous tissue and to reach the brain where its aggregation determines the fatal neuropathological issue of TSEs. ${ }^{10}$ Thus, apart from the brain in which $\operatorname{PrP}^{\mathrm{sc}}$ accumulation is a dead end, $\operatorname{PrP}^{\mathrm{sc}}$ concentration is expected to be low in other tissues or in biological samples such as blood. ${ }^{11}$

Today, v-CJD epidemics in humans have only clinically emerged in most susceptible individuals, ie with homozygotic methionine genotype in PrP 129 (MET-MET) codonknown to be associated with short incubation period-and with a window period of alimentary contamination during childhood, when factors such as intestinal barrier functionality are critical. ${ }^{12}$ Recently, a novel $\operatorname{PrP}^{\text {sc }}$ type in a patient exhibiting prion protein gene $129 \mathrm{VV}$ polymorphism was reported as atypical because of certain clinical and molecular $\operatorname{PrP}^{\text {sc }}$ features overlapping with those of $\mathrm{v}-\mathrm{CJD} .^{13}$ Nonetheless, recent studies providing evidence of 'asymptomatic infected carriers' in the UK population, as well as the fourth case of 'highly probable' transfusional cases already reported today are in favour of a possible 'underground propagation' of $\operatorname{PrP}^{\text {sc }}$ within the human population. ${ }^{14-18}$ Though its reality may be considered as evidenced, its extent and its evolution in the exposed populations, within such long delays of asymptomatic incubation and with possible lifelong asymptomatic carrier cases, are still very difficult to estimate.

Therefore, diagnostic tests for the detection of $\mathrm{PrP}^{\mathrm{sc}}$ infection at onset are required for an efficient control of this disease. In a research strategy for the definition of test principles suitable for the early detection of infection by $\operatorname{PrP}^{\mathrm{sc}}$ in humans, we have been interested in screening molecules for their ability to capture, concentrate and/or precipitate $\operatorname{PrP}^{\mathrm{sc}}$.

After having screened numerous molecules and devices for such properties, one of the selected molecules, streptomycin, is a well-known antibiotic and revealed capable of aggregating $\mathrm{PrP}^{\mathrm{sc}}$ molecules from different tissue origins with different stages of aggregation. ${ }^{19}$ Streptomycin is now used to increase the sensitivity of $\mathrm{PrP}^{\mathrm{sc}}$ immunodetection using its properties to aggregate and flocculate $\operatorname{PrP}^{s c}$ molecules in different tissues of animals challenged for experimental TSE. ${ }^{20}$ Moreover, successful precipitation of human brain $\mathrm{PrP}^{\mathrm{sc}}$ with this agent was reported in feasibility studies compared with animal samples. ${ }^{19,20}$ These properties were also used in immunohistochemistry, giving specific and sensitive immonoreactive $\operatorname{PrP}^{\text {sc }}$ deposits in both natural and experimental scrapie and BSE diagnosis. ${ }^{21,22}$

To ascertain the reliability and specificity of sample treatment with streptomycin for developing diagnostic detection of $\operatorname{Pr}^{\mathrm{sc}}$ in humans, we have designed a study using streptomycin followed by a reference western blot (WB) immunodetection on well-characterized reference human brain material from various forms of TSE in parallel with equivalent brain material from confirmed non-TSE patients. The protocol was also applied for tonsil tissue from v-CJD patients, in parallel with equivalent material from non-TSE patients. We thus present here the results obtained at first on a cohort of 150 patients from which necropsic human brain tissue was expertised for final TSE diagnosis confirmation by immunohistological and biochemical procedures. They represent 98 TSE cases and 52 non-TSE controls. Second, we present the results obtained on a small cohort of five patients from whom tonsil tissue was assessed for v-CJD diagnostic; two of them were further confirmed as v-CJD after post-mortem brain examination.

\section{MATERIALS AND METHODS Patient's Cohort}

For brain tissue, samples were obtained after necropsy of 150 patients suspected of CJD in anatomopathology laboratories of Lyon, Bordeaux and Marseille. TSE disease was confirmed in 98 patients (neuropathological lesions, immunohistochemistry and immunodetection of $\operatorname{PrP}^{\text {res }}$ by $\mathrm{WB}){ }^{23}$ For positive TSE patients, prion protein $(P R N P)$ gene mutation analysis, ${ }^{24}$ codon 129 polymorphism of the PRNP gene, ${ }^{25} \mathrm{PrP}^{\text {res }}$ molecular typing ${ }^{26}$ and aetiology are reported in Table 1. Prion disease was rejected in the remaining 52 patients. The final diagnosis of non-TSE patients was further completed: 19 Alzheimer's disease, 4 Lewy body dementia, 4 Parkinson's disease, 2 fronto-temporal dementia and 23 others types of pathologies. For tonsil tissue, samples were obtained after tonsillectomy for diagnostic purpose of suspected v-CJD in five patients. Two patients were classified $\mathrm{v}$-CJD after confirmed diagnostics carried out on brain necropsic tissues, whereas the three others were confirmed negatives for $\mathrm{v}$-CJD diagnosis.

\section{Common Pretreatment of Samples Used in Comparative Study}

To compare WB protocols on brain tissue, only available aliquot samples stored at $-80^{\circ} \mathrm{C}$ from the same brain area (prefrontal cortex) were used. In first series, $500 \mathrm{mg}$ of prefrontal cortex was homogenized with distilled water at $20 \%$. Homogenates were forced through a $0.5 \mathrm{~mm}$ diameter needle and divided into four aliquots for the different concentration protocols tested. In second series, with the same protocol, we prepared another homogenate from $20 \mathrm{mg}$ of prefrontal cortex and divided it into four aliquots. The precipitation of $\mathrm{PrP}^{\mathrm{sc}}$ was thus compared with different techniques using a small amount $(5 \mathrm{mg})$ of brain tissue.

\section{Streptomycin Protocol (Streptomycin)}

Analytical comparison of four protocols with determination of dilution limit was performed on $25 \mu \mathrm{l}$ of the $20 \%$ 
Table 1 Diagnostic classification of studied TSE patients

\begin{tabular}{|c|c|c|c|c|}
\hline \multirow{2}{*}{$\begin{array}{l}\text { No. of cases } \\
80\end{array}$} & \multirow{2}{*}{$\begin{array}{l}\text { Aetiology } \\
\text { Sporadic }\end{array}$} & \multicolumn{2}{|c|}{ PrPres molecular typing/number of samples/PRNP mutation } & \multirow{2}{*}{$\begin{array}{l}\text { Prion protein codon } \\
129 \text { polymorphism } \\
M M ; n=50 ;\end{array}$} \\
\hline & & Type $1 ; n=61$ & Type 1 & \\
\hline & & & & $\mathrm{MV} ; n=8$ \\
\hline & & & Type 1 majority+type 2 & $\mathrm{MM} ; n=3$ \\
\hline & & & & $W_{;} n=9$ \\
\hline & & & Type 2 majority+type 1 & $\mathrm{MM} ; n=1$ \\
\hline & & & & $\mathrm{W} ; n=1$ \\
\hline \multirow[t]{2}{*}{14} & Genetic & Type $1 ; n=9$ & E200K & $\mathrm{MM} ; n=6$ \\
\hline & & Type $2 ; n=2$ & D178N FFI & $\mathrm{MM} ; n=2$ \\
\hline \multirow[t]{2}{*}{2} & latrogenic: growth hormone & Type $1 ; n=2$ & & $\mathrm{MM} ; n=1$ \\
\hline & & & & $\mathrm{MV} ; n=1$ \\
\hline 2 & Variant & Type $2 \mathrm{~b} ; n=2$ & & $\mathrm{MM} ; n=2$ \\
\hline
\end{tabular}

CJD: Creutzfeldt-Jakob disease; D: aspartic acid; E: glutamic acid; FFI: fatal familial insomnia; I: isoleucine; K: lysine; M: methionine; N: asparagine; V: valine.

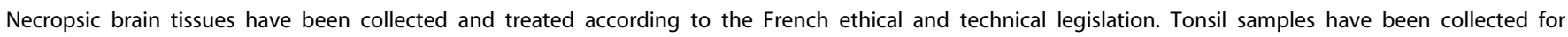
diagnostic purpose of variant CJD.

homogenate (corresponding to $5 \mathrm{mg}$ equivalent brain tissue) diluted twofold in phosphate-buffered saline (PBS) $2 \times$ ( $\mathrm{pH}=7.2$, DOC 1\%, NP-40 1\%).

For the cohort examination, $5 \mathrm{mg}$ of prefrontal cortex was directly homogenized in PBS $(\mathrm{pH}=7.2)$ DOC $0.5 \%, \mathrm{NP}-40$ $0.5 \%$ representing a final $10 \%$ brain homogenate. For tonsil tissues, $10 \%$ homogenates were simply made in $5 \%$ glucose. Tissues were all treated with $\mathrm{PK}(80 \mu \mathrm{g} / \mathrm{ml})$ for $30 \mathrm{~min}$ at $37^{\circ} \mathrm{C}$ and streptomycin $(200 \mathrm{mg} / \mathrm{ml})$ was added for $1 \mathrm{~h}$ at $37^{\circ} \mathrm{C}$ as previously described. ${ }^{19}$ Thus, after having made $10 \%$ homogenates from $5 \mathrm{mg}$ of TSE brain, we used a $0.14 \mathrm{M}$ final concentration of streptomycin in $\mathrm{PK}$ incubation medium. Samples were then submitted to centrifugation for $15 \mathrm{~min}$ at $17000 \mathrm{~g}$. A second centrifugation was made when a slightly milky zone was observed above the pellet. Pellets were resuspended in $4 \%$ SDS denaturating buffer (Tris $\mathrm{HCl} \mathrm{pH}=6.8$ $125 \mathrm{mM}$, glycerol 20\%) and boiled for $10 \mathrm{~min}$ at $100^{\circ} \mathrm{C}$. Then, they were further processed as indicated below for WB analysis.

\section{Ultracentrifugation Protocol}

For the analytical comparison of the four protocols, 500 or $25 \mu \mathrm{l}$ of the $20 \%$ homogenate (corresponding to 100 or $5 \mathrm{mg}$ equivalent brain tissue respectively) was diluted twofold in $10 \%$ glucose.

In our laboratory, this protocol is currently performed since 1998 with $100 \mathrm{mg}$ of prefrontal cortex as previously described $^{27}$ with slight modifications; prior to ultracentrifugation in Sarkosyl, homogenates were digested by proteinase $\mathrm{K}(25 \mu \mathrm{g} / \mathrm{ml})$ for $1 \mathrm{~h}$ at $37^{\circ} \mathrm{C}$ before stopping digestion by PMSF $1 \mathrm{mM}$.

\section{Sodium Phosphotungstic Acid Protocol}

For the analytical comparison of the four protocols, 250 or $25 \mu \mathrm{l}$ of the $20 \%$ homogenate (corresponding to 50 or $5 \mathrm{mg}$ equivalent brain tissue, respectively) was diluted twofold in PBS buffer $2 \times$ without $\mathrm{Ca}^{+}{ }^{+}$and $\mathrm{Mg}^{+}{ }^{+}(\mathrm{pH}=7.2)$. The protocol was strictly performed as previously described with 500 or $50 \mu \mathrm{l}$ of $10 \%$ homogenate ( 50 or $5 \mathrm{mg}$ ) of prefrontal cortex. $^{28}$

\section{Centrifugation Step Concentration Protocol (LC)}

For the analytical comparison of the four protocols, 40 or $25 \mu \mathrm{l}$ of the $20 \%$ homogenate (corresponding to 8 or $5 \mathrm{mg}$ equivalent brain tissue, respectively) was diluted twofold in Tris-buffered Saline $2 \times(\mathrm{pH}=8)$. The protocol corresponding to the 'Sample preparation II' described by Lee et al. ${ }^{29}$ was performed as previously described with 8 or $5 \mathrm{mg}$ of prefrontal cortex.

\section{WB Immunodetection}

Each sample was analysed on 12\% Nu-PAGE gels. Gels were then electroblotted on to PVDF membranes and blocked with $5 \%(\mathrm{~W} / \mathrm{V})$ milk powder in PBS for $1 \mathrm{~h}$. 


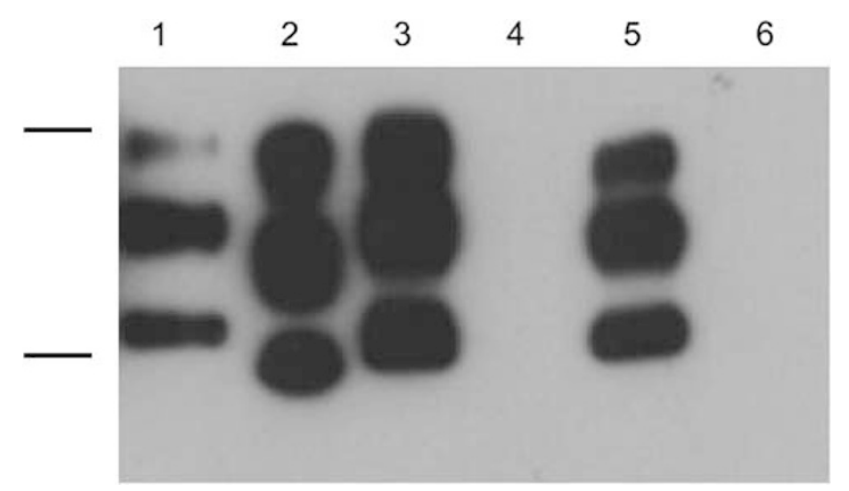

Figure 1 Analysis of supernatants after streptomycin precipitation on $5 \mathrm{mg}$ CJD brain. Immunoblot analysis of prefrontal cortex homogenates obtained from different suspected CJD patients is shown. Black lines correspond to molecular mass marker of 33 and $20 \mathrm{kDa}$, up to down, respectively. PrPres is immunodetected by 3F4 Ab. Lanes 1 and 2: PrP res types 1 and 2, respectively, extracted using ultracentrifugation protocol. Lanes 3 and 4 , and 5 and 6: PrPres type 1 from two CJD patients, extracted using streptomycin protocol, respectively pellets (lanes 3-5) and supernatants (lanes 4-6).
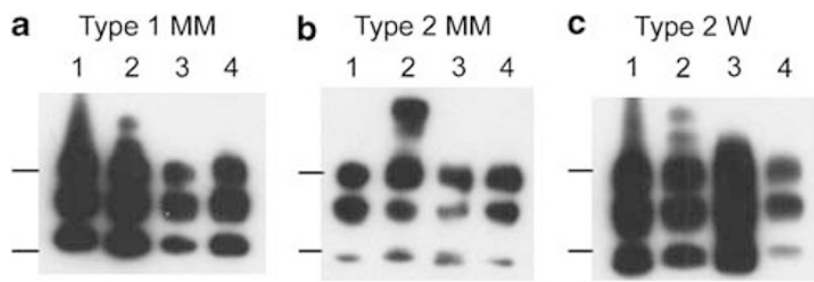

Figure 2 (a-c) Comparison of PrP ${ }^{\text {res }}$ signals after different protocols of precipitation with the recommended initial amount of tissues. Immunoblot analysis of prefrontal cortex homogenates obtained from different suspected TSE patients is shown. Black lines correspond to molecular mass marker of 33 and $20 \mathrm{kDa}$, up to down, respectively. Here, $1 \mathrm{mg}$ equivalent brain was load in each well. PrP ${ }^{\text {res }}$ is immunodetected by $3 F 4$ Ab. (a) s-CJD type 1 PrPres; (b) FFI type 2 MM PrPres and (c) s-CJD type 2 VV PrPres. Lanes 1: samples processed by the ultracentrifugation protocol (UC). Lanes 2 : samples processed by the centrifugation step concentration protocol (LC). Lanes 3: samples processed by the sodium phosphotungstic acid protocol (NaPTa). Lanes 4: samples processed by the streptomycin protocol.

Immunodetection of $\operatorname{PrP}^{\text {res }}$ was observed using 1/10000 3F4 purified anti-PrP monoclonal antibody (Proteogenics, USA) for $1 \mathrm{~h}$ at room temperature and revealed by chemiluminescence substrate West Dura (Pierce, USA).

\section{RESULTS}

\section{Analytical Features}

As shown in Figure $1, \mathrm{PrP}^{\mathrm{res}}$ signal is totally collected in the pellet as no immunodetection was evidenced in the supernatant. Thus, this concentration corresponding to $0.14 \mathrm{M}$ of streptomycin in PK digestion medium was adopted for following validation in human TSE brain and tonsil samples.

We then compared the ability of streptomycin concentration $v s$ our usual technique and $v s$ other established sensitive protocols. To observe signals on the same membrane after a Type $1 \mathrm{MM}$

$\begin{array}{llll}1 & 2 & 3 & 4\end{array}$

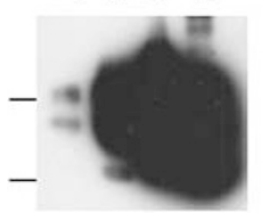

b Type $2 \mathrm{MM}$

$\begin{array}{llll}1 & 2 & 3 & 4\end{array}$

c Type $2 \mathrm{~W}$

$\begin{array}{llll}1 & 2 & 3 & 4\end{array}$
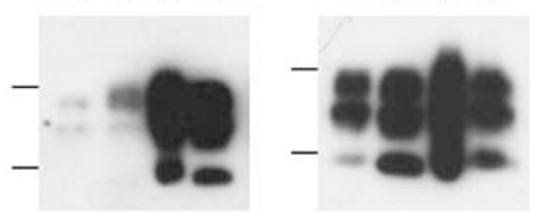

Figure 3 Comparison of PrP ${ }^{\text {res }}$ signals after performing the four protocols with initial $5 \mathrm{mg}$ of brain tissue. Immunoblot analysis of prefrontal cortex homogenates obtained from different suspected CJD patients is shown. Black lines correspond to molecular mass markers of 33 and $20 \mathrm{kDa}$, up to down, respectively. Here, $5 \mathrm{mg}$ of brain tissue was performed for each protocol and then $1 \mathrm{mg}$ equivalent brain was load in each well. PrPres is immunodetected by 3F4 Ab. (a) s-CJD Met/Met type 1 PrPres; (b) FFI Met/ Met type $2 \mathrm{PrP}^{\text {res }}$ and (c) s-CJD Val/Val type $2 \operatorname{PrP}^{\text {res }}$. Lanes 1: samples processed by the ultracentrifugation protocol (UC). Lanes 2: samples processed by the centrifugation step concentration protocol (LC). Lanes 3: samples processed by the sodium phosphotungstic acid protocol (NaPTa). Lanes 4: samples processed by the streptomycin protocol.

immunodetection, we loaded in each well $1 \mathrm{mg}$ equivalent brain tissue obtained for each of the four different methods. As shown in Figure $2 \mathrm{a}-\mathrm{c}$, the detected $\mathrm{PrP}^{\mathrm{res}}$ signals indicated that streptomycin was a pertinent agent of concentration of $\mathrm{PrP}^{\mathrm{res}}$ in the three different types of CJD samples. Indeed for the FFI Met/Met type 2 sample, signals with the four techniques were very similar, whereas for CJD Met/Met type 1 $\mathrm{PrP}^{\mathrm{res}}$, signals appeared to be slightly more important with ultracentrifugation protocol (UC) and LC protocols than with streptomycin and NaPTa protocols. Finally, for CJD Val/ Val type 2, NaPTa and UC protocols gave better $\mathrm{PrP}^{\mathrm{res}}$ signals than the two others.

In the second series, we compared the analytical sensitivity and the ability of each protocol to recover $\operatorname{PrP}^{\text {sc }}$ from only $5 \mathrm{mg}$ of brain tissue. As shown in Figure 3, NaPTa and streptomycin protocols were the most efficient procedures for good recovery of $\mathrm{PrP}^{\text {res }}$ in s-CJD Met/Met 1 (Figure 3a) and genetic CJD Met/Met 2 (Figure 3b), whereas no significant difference was seen for s-CJD Val/Val 2 samples (Figure 3c). For s-CJD Met/Met 1, we observed that UC protocol (Figure 3a, lane 1) was less sensitive, leading only very faint $\mathrm{PrP}^{\text {res }}$ signal on overexposed WB. Dilution studies on s-CJD Met/ Met 1 and Val/Val 2 permitted to confirm that $\mathrm{NaPTa}$ and streptomycin were the most sensitive protocols (data not shown). For FFI Met/Met 2 samples, serial dilutions of 5-, 10and 20-fold were performed, corresponding to $0.2,0.10$ and $0.05 \mathrm{mg}$ of tissue loaded on gel. As shown in Figure 4, we also observed that $\mathrm{NaPTa}$ and streptomycin were the most sensitive protocols, whereas the two others were not able to detect $\operatorname{PrP}^{\text {res }}$ in these diluted samples. We could also confirm that, in the cases of s-CJD Met/Met 1, s-CJD Val/Val 2 and FFI Met/Met 2, the dilution limit differed as expected from known variation of $\operatorname{PrP}^{\mathrm{sc}}$ concentration in corresponding samples. 


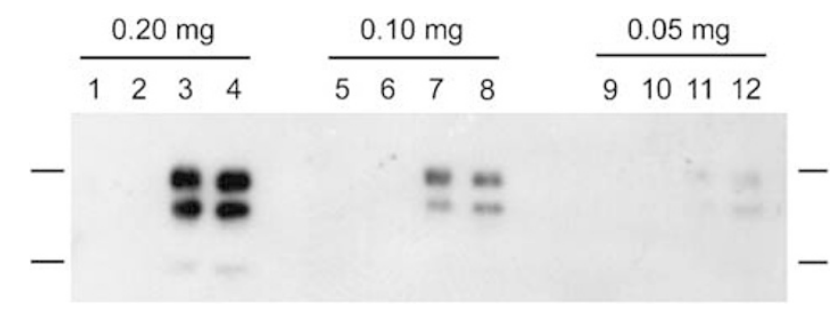

Figure 4 Detection of PrPres after dilution of type 2 Met/Met FFI sample. Immunoblot analysis of prefrontal cortex homogenates obtained from one genetic (TSE) patient with FFI is shown. The sample of FFI type 2 Met/Met $\mathrm{PrP}^{\text {res }}$ was serially diluted to obtain $0.20 \mathrm{mg}$ (lanes $1-4$ ); $0.10 \mathrm{mg}$ (lanes 5-8) and $0.05 \mathrm{mg}$ (lanes 9-12) of brain tissue. Lanes 1, 5 and 9: samples processed by the ultracentrifugation protocol (UC). Lanes 2, 6 and 10: samples processed by the centrifugation step concentration protocol (LC). Lanes 3, 7 and 11: samples processed by the sodium phosphotungstic acid protocol ( $\mathrm{NaPTa}$ ). Lanes 4, 8 and 12: samples processed by the streptomycin protocol.

\section{Cerebral PrPres Detection Using Streptomycin Precipitation}

All brain specimens from the 98 confirmed TSE patients showed $\mathrm{PrP}^{\text {res }}$-positive signal on $\mathrm{WB}$ by immunodetection with 3F4 monoclonal antibody after streptomycin precipitation. As represented in Figure 5, the three-band $\operatorname{Pr}^{\mathrm{res}}$ pattern is clearly identified for these samples. When the signal was saturated, a smear all along the lane was found. For the specimen of the 52 non-TSE patients (examples in Figure 5), no signal was detected indicating that (i) the digestion of $\mathrm{PrP}^{\mathrm{c}}$ by $\mathrm{PK}$ was complete, and (ii) streptomycin addition did not interfere with immunodetection.

Most importantly, TSE and non-TSE patients were correctly classified by the assay leading to specificity and sensitivity at $100 \%$.

\section{Cerebral PrPres Molecular Typing Using Streptomycin Precipitation}

The evaluation of streptomycin influence on the $\mathrm{PrP}^{\mathrm{res}}$ molecular typing was further analysed. Using streptomycin precipitation, we were able to detect the two major WB bands with distinct apparent molecular weights of unglycosylated isoform that characterized types 1 and $2 \mathrm{PrP}^{\mathrm{res}}$. So types 1 and $2 \mathrm{PrP}^{\text {res }}$ were clearly discriminated. Although we did not perform exact determination of the ratio diglycosylated/ monoglycosylated bands, we nevertheless examine $\operatorname{PrP}^{\text {res }}$ molecular pattern obtained on different brain area and brain extracts in different experiments with streptomycin precipitation. The characteristic v-CJD glycotype in type $2 \mathrm{~b}$ $\mathrm{PrP}^{\mathrm{res}}$ seemed to be conserved (Figure 6, lane 6) and was also easily discriminated from those of sporadic type $2 \mathrm{a}$ (Figure 6 , lane 4) and moreover, from type $1 \mathrm{PrP}^{\text {res }}$ (Figure 6, lanes 3 and 5). Concerning type $1 \mathrm{PrP}^{\text {res }}$, characteristic molecular patterns for sporadic (Figure 6, lane 3) and E200K PRNP mutation (Figure 6, lane 5) are clearly distinguishable too.

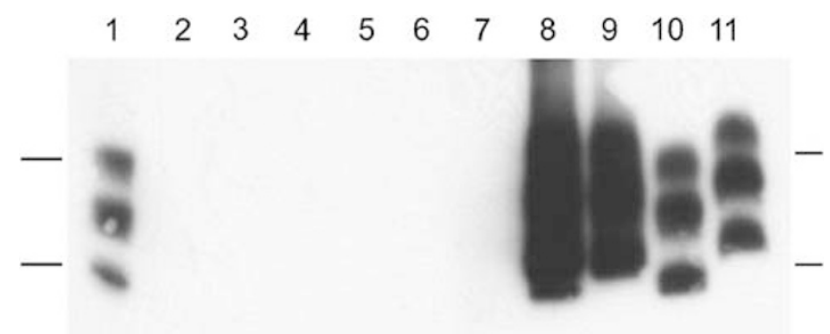

Figure 5 Analysis of brain PrP ${ }^{\text {res }}$ of suspected CJD patients using streptomycin precipitation (representative selection). Immunoblot analysis of prefrontal cortex homogenates obtained from different suspected CJD patients is shown. Black lines correspond to molecular mass markers of 33 and $20 \mathrm{kDa}$, up to down, respectively. Lane 1: control CJD PrP ${ }^{\text {res }}$ type 2 (ultracentrifugation protocol); Lanes 2-7: non-CJD patients; Lanes 8-11: CJD patients.

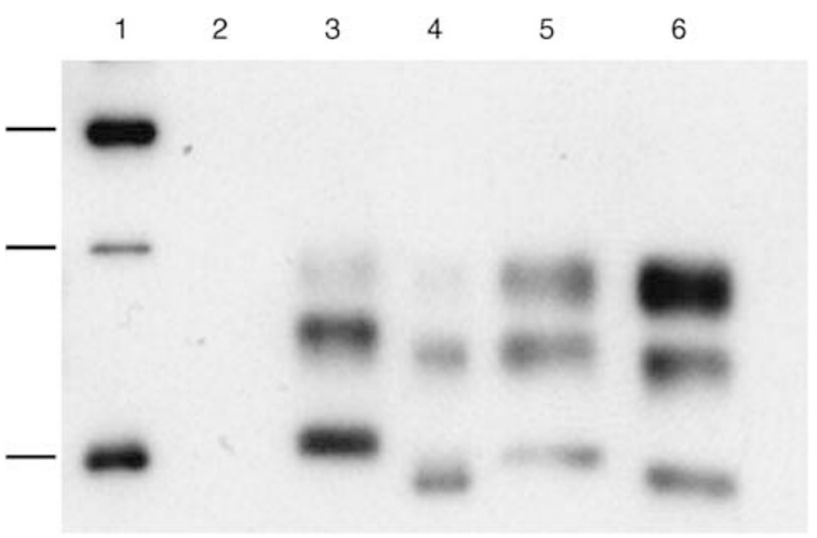

Figure 6 Distinction of PrP res types 1 and 2 using streptomycin protocol. Immunoblot analysis of prefrontal cortex homogenates obtained from different CJD patients is shown. Black lines correspond to molecular mass markers of 40,30 and $20 \mathrm{kDa}$, up to down, respectively. PrPres is immunodetected by 3F4 Ab. Lane 1: molecular markers, respectively from up to down: 40,30 and $20 \mathrm{kDa}$. Lane 2: non-CJD control sample. Lane 3: type $1 \mathrm{PrP}^{\text {res }}$ (sporadic CJD patient); lane 4: type 2a $\operatorname{PrP}^{\text {res }}$ (sporadic CJD patient); lane 5: type $1 \mathrm{PrP}^{\text {res }}$ (genetic CJD: E200K PRNP mutation); lane 6: type $2 \mathrm{~b} \operatorname{PrP}^{\text {res }}$ ( $v$-CJD patient).

\section{Tonsil Examination for PrP ${ }^{\text {res }}$ Detection with Streptomycin Protocol}

The type $2 \mathrm{~b} \operatorname{PrP}^{\mathrm{res}}$ was clearly identified in the two tonsils extracted from confirmed v-CJD cases (Figure 7, lanes 4 and 5) with the characteristic three-band pattern, whereas the three non-v-CJD showed a background and nonspecific signal around $30 \mathrm{kDa}$ that did not fit with any characteristic three-band pattern of $\operatorname{PrP}^{\text {res }}$ (Figure 7, lanes 1-3). In v-CJD tonsils, the relative ratio of glycotype appeared to be consistent with that of corresponding brain extract.

\section{DISCUSSION}

The streptomycin precipitation protocol performed in this study is adapted from the one reported for brain $\operatorname{PrP}^{\mathrm{sc}}$ detection in natural scrapie, natural BSE and experimental 


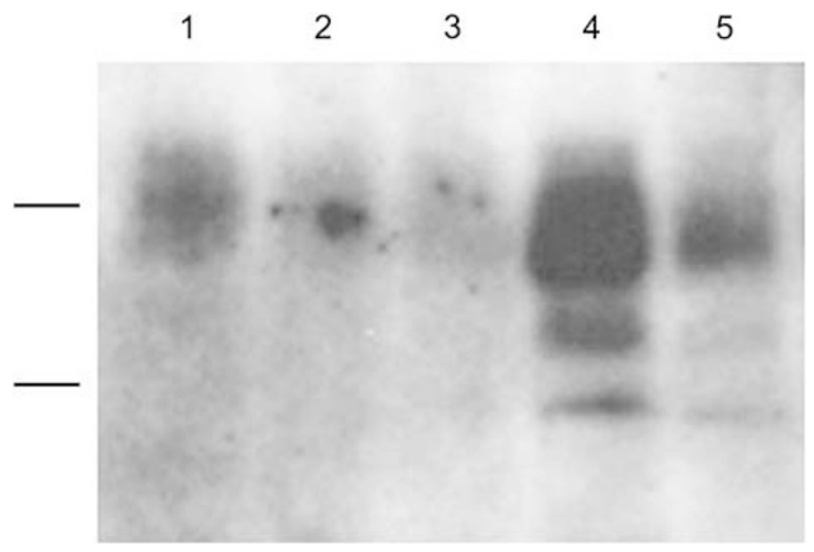

Figure 7 Tonsil analysis for PrP ${ }^{\text {res }}$ detection in five suspected v-CJD cases using streptomycin protocol. Immunoblot analysis of tonsil homogenates obtained from five suspected v-CJD patients is shown. Black lines correspond to molecular mass markers of 30 and $20 \mathrm{kDa}$, up to down, respectively. PrPres is immunodetected by $3 \mathrm{~F} 4 \mathrm{Ab}$. Lanes 1-3: non-v-CJD patients' tonsil examination using streptomycin protocol. Lanes 4 and 5 : v-CJD patients' tonsil examination using streptomycin protocol.

BSE in mice. ${ }^{19}$ Analysis of the supernatants after precipitation from a very low amount of human brain $(5 \mathrm{mg})$ compared with that routinely used for UC $(100 \mathrm{mg})$ shows that all $\mathrm{PrP}^{\text {res }}$-specific signal detected can be concentrated in the pellet, without significant residual signal in supernatant. So the full process used in animal TSE was successfully transferred to human application without modification.

Although the main objective was not a complete analytical study, we nonetheless compared this protocol with different reported processes using concentration steps as UC commonly used in our lab for CJD diagnosis, ${ }^{27}$ a centrifugation protocol (LC) ${ }^{29}$ and NaPTa step precipitation $(\mathrm{NaPTa})^{28}$ as previously described by others. In first series, these concentration protocols were tested as reported without modification prior to a unique $\mathrm{WB}$ analysis. Here, the amounts of tissue tested were those recommended for streptomycin, LC, NaPTa and UC protocols $(5,8,50$ and $100 \mathrm{mg}$, respectively). Thus, comparison of an equivalent quantity was achieved in $\mathrm{WB}$ analysis by loading $1 \mathrm{mg}$ of brain tissue extract into the gel. In second series, we have compared the ability of each protocol to recover $\mathrm{PrP}^{\text {res }}$ from a small amount of brain tissue equivalent to that processed in streptomycin protocol $(5 \mathrm{mg})$. This reproduced situations when only small quantities of original sample material are available (ie tonsils).

From the present study, advantages and drawbacks of each protocol can be rapidly listed. Elimination of most of soluble proteins by UC allows loading high amounts of tissue (20-50 mg) without unspecific noise (not shown) but it is (i) time consuming ( $4 \mathrm{~h} 35 \mathrm{~min}$ ), (ii) large serial analysis cannot be performed within 1 day and (iii) recovery of the pellet needs experience with an important impact of manipulators' skill (particularly when initial quantity is below $10 \mathrm{mg}$ of tissue). Moreover, this technique revealed poor sensitivity for recovering $\mathrm{PrP}^{\text {res }}$ when performed from only $5 \mathrm{mg}$ of tissue, in particular for s-CJD Met/Met 1 and FFI Met/Met 2. It thus appeared to be extremely dependent upon the initial amount of tissue. With NaPTa, high recovery of $\mathrm{PrP}^{\text {res }}$ in the pellet vs $\operatorname{PrP}^{\mathrm{c}}$ offers a potential to load high amounts of tissue (25-50 mg) without unspecific noise but requires (i) long sequential steps ( $3 \mathrm{~h} 30 \mathrm{~min}$ ), (ii) time-consuming buffer and stock solution preparation and (iii) frequent need to solubilize a white precipitate as reported. ${ }^{28}$ Nonetheless, NaPTa also succeeded in recovering $\operatorname{PrP}^{\text {res }}$ in the different $\operatorname{PrP}^{\mathrm{sc}}$ strains tested here from $5 \mathrm{mg}$ of brain tissue. Using the centrifugation method (LC), ${ }^{29}$ centrifugation at $20000 \mathrm{~g}$ reduced the preparation time $(3 \mathrm{~h} 05 \mathrm{~min})$ whatever the amount of tissue. However, with the recommended initial amount of $8 \mathrm{mg}$ of tissue, $\mathrm{PrP}^{\mathrm{res}}$ was clearly recovered, but when decreased to only $5 \mathrm{mg}$, the sensitivity dropped. For LC, ratio of proteinase $\mathrm{K} / \mathrm{amount}$ of brain tissue is the highest of the four techniques, still proteinase $\mathrm{K}$ concentration needed to be increased to completely digest $\operatorname{PrP}^{\mathrm{c}}$ for few cases. Finally, streptomycin concentration gave specific results with good sensitivity, with the lowest amount of tissue and the shortest preparation time $(2 \mathrm{~h} 30 \mathrm{~min})$. Only in few cases complete recovery of pellet required a second low-speed and short centrifugation step. In dilution experiments, the most sensitive protocols were found to be NaPTa and streptomycin.

The main objective was thereafter to evaluate the sensitivity and specificity of the streptomycin protocol in a cohort representing the diversity of human TSE types.

The results obtained here with the post-mortem diagnosed cohort of 98 TSE patients and 52 non-TSE patients yielded $100 \%$ specificity and $100 \%$ sensitivity, showing neither false positive nor false negative. This performance with such specificity provides criteria enabling the use of this new protocol for $\operatorname{PrP}^{\text {res }}$ concentration. Indeed, no false negative could be admissible in the field of human TSE diagnosis. Actual sensitivity of the protocol also complies with the objective of detecting brain $\operatorname{PrP}^{\text {res }}$, for post-mortem diagnostic purposes. Thus, precipitation by streptomycin was found to be helpful in terms of practicability, and could replace a cumbersome UC method with its different drawbacks. This protocol gave similar signal than the two other methods used, ie NaPTa and LC, when the same quantities of brain tissue are loaded in the gel. For post-mortem brain diagnosis, a decrease in the amount of tissue is not a critical step. But for ante-mortem biopsies of tonsils and other tissues as reported for olfactory mucosa and in our previous study on peripheral nerves, ${ }^{27,30}$ processing low amounts without a good recovery of $\operatorname{PrP}^{\mathrm{sc}}$ would be a limitation for certain methods. In the case of tonsil examination in clinically suspected v-CJD cases, our results obtained with streptomycin permitted to clearly detect characteristic $\mathrm{PrP}^{\text {res }}$ pattern in this lymphoid tissue. Even if a faint signal can be detected around $30 \mathrm{kDa}$ on non-v-CJD tonsil samples, no 
characteristic pattern of $\mathrm{PrP}^{\mathrm{res}}$ can be detected on overexposed WB with these negative samples, whereas it clearly exists for positive samples (data not shown). We have noticed incidentally that freezing-thawing cycles have decreased this nonspecific signal, whereas increasing PK concentration up to twofold had no influence. Some optimization should be performed to improve specificity if single band analysis ever became relevant, but this streptomycin protocol should be sufficient for a global rapid test. These results were correlated with the confirmed diagnosis obtained after brain examination, confirming again a good sensitivity and specificity. These data, as those recently reported in C57Bl/6 mice inoculated i.c. or i.p., provide evidence that $\mathrm{PrP}^{\mathrm{res}}$ could be detected either in lymphoid organs or in brain with a good sensitivity. ${ }^{31}$ Streptomycin seems then to be an efficient tool for peripheral tissue examination in living individuals.

The present protocol permits the recognition of the different glycoforms of human brain $\mathrm{PrP}^{\text {res }}$ by $3 \mathrm{~F} 4$ antibody with the expected shift in the molecular weight. ${ }^{19}$ This shift does not alter the classical three-band pattern and therefore, this method complies with the molecular $\operatorname{PrP}^{\text {res }}$ typing field. ${ }^{23}$ Finally, v-CJD molecular $\operatorname{PrP}^{\text {res }}$ pattern is discriminated from those found in sporadic or genetic CJD. Indeed, in our present conditions, we have worked with streptomycin quantity within the range of those shown not to increase molecular weight patterns in precipitated material from PK-digested homogenate. ${ }^{19}$ Moreover, in another study, ${ }^{20}$ still different patterns were reported using protocols inverting the proteinase $\mathrm{K}$ and streptomycin incubation steps when compared with our protocol. They thus yielded patterns diverging from our own results, which we have obtained after setting up conditions most appropriate for our study dedicated to human CJD diagnostics. Thus our analyses had to be comparable in routine conditions to other reference techniques used in the human medical domain.

In conclusion, it is the first report to our knowledge on streptomycin WB for routine diagnosis of human TSEs. Streptomycin seems to act as a good precipitation agent for low amount of tissue containing $\operatorname{PrP}^{\mathrm{sc}}$ and thus permits quick analyses on small quantities of tissues, with particular interest for biopsies. This protocol allowed us to detect $\mathrm{PrP}^{\mathrm{res}}$ in small amounts of tonsil tissue for v-CJD patients. For peroneal nerve analysis in s-CJD as already reported, ${ }^{27,32}$ it could also be an alternative to UC as only $20 \%$ of sensitivity was found. Streptomycin also seems to be a valid alternative protocol to NaPTa when small amounts of tissue are available. Because satisfactory recovery efficiency of $\mathrm{PrP}^{\text {res }}$ spiked in CSF and urine was recently claimed, ${ }^{20}$ it could now be evaluated as a tool for a test detecting $\operatorname{PrP}^{\text {sc }}$ in human and animal biological fluids. Finally, because streptomycin is a well-known antibiotic available for human therapy, these in vitro results could raise questions about its eventual effects in vivo.

\section{DISCLOSURE/CONFLICT OF INTEREST}

All authors but two are hired by non-profit organizations and/or University Hospitals and declare no conflict of interest. The two authors from

BioMérieux also declare no conflict of interest as HP has left this company and $\mathrm{MD}$ is no longer involved in Prion Diagnostics, as this R\&D domain has been closed by BioMérieux.

1. Prusiner SB. Prions. Proc Natl Acad Sci USA 1998;95:13363-13383.

2. Collins SJ, Lawson VA, Masters CL. Transmissible spongiform encephalopathies. Lancet 2004;363:51-61.

3. Hsiao K, Baker HF, Crow TJ, et al. Linkage of a prion protein missense variant to Gerstmann-Straussler syndrome. Nature 1989;338:342-345.

4. Gambetti $P$, Parchi $P$, Petersen RB, et al. Fatal familial insomnia and familial Creutzfeldt-Jakob disease: clinical, pathological and molecular features. Brain Pathol 1995;5:43-51.

5. Chazot G, Broussolle E, Lapras C, et al. New variant of CreutzfeldtJakob disease in a 26-year-old French man. Lancet 1996;347:1181.

6. Will RG, Ironside JW, Zeidler $M$, et al. A new variant of CreutzfeldtJakob disease in the UK. Lancet 1996;347:921-925.

7. Bruce ME, Will RG, Ironside JW, et al. Transmissions to mice indicate that 'new variant' CJD is caused by the BSE agent. Nature 1997;389:498-501.

8. Hill AF, Desbruslais $M$, Joiner $S$, et al. The same prion strain causes vCJD and BSE. Nature 1997;389:448-450, 526.

9. Collinge J, Whitfield J, McKintosh E, et al. Kuru in the 21st century-an acquired human prion disease with very long incubation periods. Lancet 2006;367:2068-2074.

10. Glatzel M, Giger O, Braun N, et al. The peripheral nervous system and the pathogenesis of prion diseases. Curr Mol Med 2004;4:355-359.

11. Brown P. Blood infectivity, processing and screening tests in transmissible spongiform encephalopathy. Vox Sang 2005;89:63-70.

12. Boelle PY, Cesbron JY, Valleron AJ. Epidemiological evidence of higher susceptibility to vCJD in the young. BMC Infect Dis 2004;4:26.

13. Mead S, Joiner S, Desbruslais $M$, et al. Creutzfeldt-Jakob disease, prion protein gene codon 129VV, and a novel PrPSc type in a young British woman. Arch Neurol 2007;64:1780-1784.

14. Bishop MT, Hart P, Aitchison L, et al. Predicting susceptibility and incubation time of human-to-human transmission of vCJD. Lancet Neurol 2006;5:393-398.

15. Llewelyn CA, Hewitt PE, Knight RS, et al. Possible transmission of variant Creutzfeldt-Jakob disease by blood transfusion. Lancet 2004;363:417-421.

16. Peden $A H$, Head MW, Ritchie DL, et al. Preclinical vCJD after blood transfusion in a PRNP codon 129 heterozygous patient. Lancet 2004;364:527-529.

17. Wroe SJ, Pal S, Siddique D, et al. Clinical presentation and pre-mortem diagnosis of variant Creutzfeldt-Jakob disease associated with blood transfusion: a case report. Lancet 2006;368:2061-2067.

18. Health_Protection_Agency. Fourth case of variant CJD infection associated with blood transfusion. (Press release) United Kingdom: Health Protection Agency; 2007 (updated 2007; cited 18 January 2007); Available from: http://www.hpa.org.uk/webw/HPAweb\&HPAweb Standard/HPAweb_C/1195733711457?p = 1171991026241.

19. Moussa A, Coleman AW, Bencsik A, et al. Use of streptomycin for precipitation and detection of proteinase $\mathrm{K}$ resistant prion protein (PrPsc) in biological samples. Chem Commun (Camb) 2006;7:973-975.

20. Dong CF, Huang YX, An R, et al. Sensitive detection of PrPSc by western blot assay based on streptomycin sulphate precipitation. Zoonoses Public Health 2007;54:328-336.

21. Bencsik AA, Coleman AW, Debeer SO, et al. Amplified immunohistochemical detection of PrPsc in animal transmissible spongiform encephalopathies using streptomycin. J Histochem Cytochem 2006;54:849-853.

22. Bencsik $A A$, Leclere $E$, Perron $H$, et al. New insights into early sequential PrPsc accumulation in scrapie infected mouse brain evidenced by the use of streptomycin sulfate. Histochem Cell Biol 2008;129:643-650.

23. WHO. WHO Manual for Surveillance of Human Transmissible Spongiform Encephalopathies, Including Variant Creutzfeldt-Jakob Disease. World Health Organization: Geneva, Switzerland, 2003, p 90.

24. Peoc'h K, Manivet $P$, Beaudry $P$, et al. Identification of three novel mutations (E196K, V203I, E211Q) in the prion protein gene (PRNP) in 
inherited prion diseases with Creutzfeldt-Jakob disease phenotype. Hum Mutat 2000;15:482.

25. Zimmermann K, Turecek PL, Schwarz HP. Genotyping of the prion protein gene at codon 129. Acta Neuropathol 1999;97:355-358.

26. Parchi P, Castellani R, Capellari S, et al. Molecular basis of phenotypic variability in sporadic Creutzfeldt-Jakob disease. Ann Neurol 1996;39:767-778.

27. Favereaux A, Quadrio I, Vital C, et al. Pathologic prion protein spreading in the peripheral nervous system of a patient with sporadic Creutzfeldt-Jakob disease. Arch Neurol 2004;61:747-750.

28. Wadsworth JD, Joiner S, Hill AF, et al. Tissue distribution of protease resistant prion protein in variant Creutzfeldt-Jakob disease using a highly sensitive immunoblotting assay. Lancet 2001;358:171-180.
29. Lee DC, Stenland CJ, Hartwell RC, et al. Monitoring plasma processing steps with a sensitive western blot assay for the detection of the prion protein. J Virol Methods 2000;84:77-89.

30. Tabaton M, Monaco S, Cordone MP, et al. Prion deposition in olfactory biopsy of sporadic Creutzfeldt-Jakob disease. Ann Neurol 2004;55:294-296.

31. Leclere E, Moussa A, Chouaf-Lakhdar L, et al. Prion early kinetics revisited using a streptomycin-based $\operatorname{PrP}($ res) extraction method. Biochem Biophys Res Commun 2008;372:429-433.

32. Favereaux A, Quadrio I, Perret-Liaudet A, et al. Prion protein accumulation involving the peripheral nervous system in a sporadic case of Creutzfeldt-Jakob disease. Neuropathol Appl Neurobiol 2003;29:602-605. 